\title{
Some generalized bivariate Bernstein operators
}

\author{
Dan Bărbosu
}




\title{
SOME GENERALIZED BIVARIATE BERNSTEIN OPERATORS
}

\author{
DAN BARBOSU \\ Department of Mathematics and Computer Sciences, North University of Baia Mare \\ Baia Mare Victoriei 76, Romania \\ dbarbosu@univer.ubm.ro
}

[Received July 22, 1999]

\begin{abstract}
A bivariate operator with knots which form a geometric progression is constructed and some approximation properties of this operator are established.

Mathematical Subject Classification: $41 \mathrm{~A} 10$

Keywords: Bernstein operator, bivariate function, parametric extensions, Korovkin theorem
\end{abstract}

\section{Preliminaries}

It is well known that the classical Bernstein operator associates the polynomial

$$
B_{n}(f ; x)=\sum_{r=0}^{n}\left(\begin{array}{c}
n \\
r
\end{array}\right) x^{r}(1-x)^{n-r} f\left(\frac{r}{n}\right)
$$

to any function $f \in C[0,1]$.

In (1.1), the approximated function $f$ is evaluated at equally spaced intervals. Further were given extensions of this operator to the case of multivariate functions by D. D. Stancu $[10,11,12]$, E. Dobrescu and I. Matei [6], C. Badea, I. Badea and H. H. Gonska [1], and D. Barbosu [2, 3, 4]. In all these extensions, the approximated function $f:[0,1]^{n} \rightarrow \mathbf{R}$ is evaluated at equally spaced intervals.

G. M. Phillips $[7,8,9]$ constructed a generalization of the classical operator of Bernstein where the approximated function $f$ is evaluated at intervals which form a geometric progression. We start by recalling some results due to G. M. Phillips containing $q$-integers.

Let $q>0$ be any positive real number. For any non-negative integer $i$ the so called $" q$-integer" was introduced, denoted by [i] and defined by

$$
[i]=\left\{\begin{array}{cc}
\frac{1-q^{i}}{1-q}, & q \neq 1 \\
i, & q=1 .
\end{array}\right.
$$

A " $q$-factorial" was defined in the following obvious way:

$$
[i] !=\left\{\begin{array}{c}
{[i][i-1] \ldots[1], \quad i=1,2, \ldots} \\
1, \quad i=0
\end{array}\right.
$$


and a " $q$-binomial coefficient" was defined as:

$$
\left[\begin{array}{l}
k \\
r
\end{array}\right]=\frac{[k] !}{[r] ![k-r] !}
$$

Note that the $q$-binomial coefficients satisfy - see [7] - the recurrence relations

$$
\left[\begin{array}{c}
k+1 \\
r
\end{array}\right]=q^{k-r+1}\left[\begin{array}{c}
k \\
r-1
\end{array}\right]+\left[\begin{array}{l}
k \\
r
\end{array}\right]
$$

and

$$
\left[\begin{array}{c}
k+1 \\
r
\end{array}\right]=\left[\begin{array}{c}
k \\
r-1
\end{array}\right]+q^{r}\left[\begin{array}{l}
k \\
r
\end{array}\right]
$$

Next, a sequence of positive linear operators $B_{n}: C[0,1] \rightarrow C[0,1]$ was constructed, which associates for any positive integer $n$ to any $f \in C[0,1]$ the polynomial

$$
B_{n}(f ; x)=\sum_{r=0}^{n} f_{r}\left[\begin{array}{c}
n \\
r
\end{array}\right] x^{r} \prod_{s=0}^{n-r-1}\left(1-q^{s} x\right) .
$$

In (1.9) an empty product denotes 1 and $f_{r}=f\left(\frac{[r]}{[n]}\right)$. Clearly, if $q=1$ the operator (1.9) is reduced to the classical Bernstein operator.

It was proved ([7], [8], [9] ) that the properties of the generalized Bernstein operator are similar to the properties of the classical Bernstein operator (1.1).

The aim of the present paper is to extend the operator (1.9) to the case of bivariate functions.

\section{Main results}

Let $I^{2}=[0,1] \times[0,1]$ be the unit square and $\mathbf{R}^{I^{2}}=\left\{f \mid f: I^{2} \rightarrow \mathbf{R}\right\}$ denote the space of real bivariate functions defined on this square.

For any function $f \in \mathbf{R}^{I^{2}}$ and any positive real numbers $q_{1}, q_{2}>0$ one denotes by

$$
B_{n_{1}}^{x}(f ; x, y)=\sum_{r_{1}=0}^{n_{1}} f_{r_{1}}\left[\begin{array}{c}
n_{1} \\
r_{1}
\end{array}\right] x^{r_{1}} \prod_{s_{1}=0}^{n_{1}-r_{1}-1}\left(1-q_{1}^{s_{1}} x\right)
$$

and

$$
B_{n_{2}}^{y}(f ; x, y)=\sum_{r_{2}=0}^{n_{2}} f_{r_{2}}\left[\begin{array}{c}
n_{2} \\
r_{2}
\end{array}\right] y^{r_{2}} \prod_{s_{2}=0}^{n_{2}-r_{2}-1}\left(1-q_{2}^{s_{2}} y\right)
$$

respectively, the parametric extensions of the operator (1.9). Note that in (2.1) and (2.2) an empty product denotes 1 and $f_{r_{1}}=f\left(\frac{\left[r_{1}\right]}{\left[n_{1}\right]}, y\right), \quad f_{r_{2}}=f\left(x, \frac{\left[r_{2}\right]}{\left[n_{2}\right]}\right)$. Clearly, 
for $q_{1}=1$ and $q_{2}=1$ the parametric extensions (2.1) and (2.2) are reduced to the parametric extensions of the classical Bernstein operator.

From the definitions (2.1) and (2.2), it follows

Lemma 2.1. The parametric extensions (2.1) and (2.2) of the operator (1.9) are linear positive operators on $C\left(I^{2}\right)$.

Lemma 2.2. Let $f \in C\left(I^{2}\right)$. Then, for any $y \in[0,1]$ the operator (2.1) has the following interpolation properties

(i) $B_{n_{1}}^{x}(f ; 0, y)=f(0, y)$

(ii) $B_{n_{2}}^{x}(f ; 1, y)=f(1, y)$.

Proof.

(i) By the definition (2.1), we obtain:

$$
\begin{gathered}
B_{n_{1}}^{x}(f ; x, y)=\sum_{r_{1}=0}^{n_{1}} f_{r_{1}}\left[\begin{array}{c}
n_{1} \\
r_{1}
\end{array}\right] x \prod_{r_{1}=0}^{r_{1}}\left(1-q_{1}^{n_{1}} x\right)= \\
=f_{0}\left[\begin{array}{c}
n \\
0
\end{array}\right] x \prod_{s_{1}=0}^{n_{1}-1}\left(1-q_{1}^{s_{1}} x\right)+f_{1}\left[\begin{array}{c}
n_{1} \\
1
\end{array}\right] x \prod_{s=0}^{n_{1}-2}\left(1-q_{1}^{s_{1}} x\right)+\ldots+ \\
+\ldots+f_{n}\left[\begin{array}{c}
n \\
n
\end{array}\right] x^{n} .
\end{gathered}
$$

Here $f_{i}=f\left(\left[\frac{i}{n}\right] y\right), i=\overline{0, n}$. Substituting $x=0$, we get that

$$
B_{n_{1}}^{x}(f ; 0, y)=f_{0}\left[\begin{array}{c}
n \\
0
\end{array}\right]=f\left(\frac{[0]}{[n]}, y\right)=f(0, y),
$$

for any $y \in[0,1]$.

(ii) Substituting $x=1$ in (2.3), one obtains $B_{n_{1}}^{x}(f ; 1, y)=f(1, y)$, for any $y \in[0,1]$.

In a similar way, we obtain

Lemma 2.3. For any $x \in[0,1]$ and any $f \in C\left(I^{2}\right)$ the operator (2.2) has the following interpolation properties

(i) $\quad B_{n 2}^{y}(f ; x, 0)=f(x, 0)$

(ii) $\quad B_{n_{2}}^{y}(f ; x, 1)=f(x, 1)$.

Lemma 2.4. The operators $B_{n_{1}}^{x}, B_{n_{2}}^{y}$ commute on $C\left(I^{2}\right)$. Their product is the linear positive operator $B_{n_{1}, n_{2}}: C\left(I^{2}\right) \stackrel{\rightarrow}{\rightarrow} C\left(I^{2}\right)$, which associates to any function $f \in C\left(I^{2}\right)$ the approximant

$$
\begin{gathered}
B_{n_{1}, n_{2}}(f ; x, y)=\sum_{r_{1}=0}^{n_{1}} \sum_{r_{2}=0}^{n_{2}} f_{r_{1}, r_{2}}\left[\begin{array}{c}
n_{1} \\
r_{1}
\end{array}\right]\left[\begin{array}{l}
n_{2} \\
r_{2}
\end{array}\right] x^{r_{1}} y^{r_{2}} \\
\prod_{s_{1}=0}^{n_{1}-r_{1}-1}\left(1-q_{1}^{s_{1}} x\right) \prod_{s_{2}=0}^{n_{2}-r_{2}-1}\left(1-q_{2}^{s_{2}} y\right) .
\end{gathered}
$$


Proof. By direct computation one obtains

$$
\begin{gathered}
B_{n_{1}}^{x} B_{n_{2}}^{y}(f ; x, y)=B_{n_{1}}^{x}\left(B_{n_{2}}^{y}(f ; x, y)\right)= \\
=B_{n_{1}}^{x}\left(\sum_{r_{2}=0}^{n_{2}} f_{r_{2}}\left[\begin{array}{c}
n_{2} \\
r_{2}
\end{array}\right] y^{r_{2}} \prod_{s_{1}=0}^{n_{2}-r_{2}-1}\left(1-q_{2}^{s_{2}} y\right)\right)= \\
=\sum_{r_{2}=0}^{n_{2}}\left[\begin{array}{c}
n_{2} \\
r_{2}
\end{array}\right] y^{r_{2}} \prod_{s_{2}=0}^{n_{2}-r_{2}-1}\left(1-q_{2}^{s_{2}} y\right) B_{n_{1}}^{x}\left(f_{r_{2}}\right)=\sum_{r_{2}=0}^{n_{2}}\left[\begin{array}{c}
n_{2} \\
r_{2}
\end{array}\right] y^{r_{2}} \prod_{s_{2}=0}^{n_{2}-r_{2}-1}\left(1-q_{2}^{s_{2}} y\right) \\
\left(\sum_{r_{1}=0}^{n_{1}}\left[\begin{array}{c}
n_{1} \\
r_{1}
\end{array}\right] x^{r_{1}} f_{r_{1}}, r_{2} \prod_{s_{1}=0}^{n_{1}-r_{1}-1}\left(1-q_{1}^{s_{1}} x\right)\right)= \\
=\sum_{r_{1}=0}^{n_{1}} \sum_{r_{2}=0}^{n_{2}}\left[\begin{array}{c}
n_{1} \\
r_{1}
\end{array}\right]\left[\begin{array}{c}
n_{2} \\
r_{2}
\end{array}\right] x^{r_{1}} y^{r_{2}} f_{r_{1}}, r_{2} \prod_{s_{1}=0}^{n_{1}-1}\left(1-q_{1}^{s_{1}} x\right) \prod_{s_{2}=0}^{n_{2}-r_{2}-1}\left(1-q_{2}^{s_{2}} y\right) .
\end{gathered}
$$

In a similar way, it follows

$$
\begin{aligned}
B_{n_{2}}^{y} B_{n_{1}}^{x}(f ; x, y)= & \sum_{r_{1}=0}^{n_{1}} \sum_{r_{2}=0}^{n_{2}}\left[\begin{array}{c}
n_{1} \\
r_{1}
\end{array}\right]\left[\begin{array}{c}
n_{2} \\
r_{2}
\end{array}\right] x^{r_{1}} y^{r_{2}} f_{r_{1}}, r_{2} \times \\
& \prod_{s_{1}=0}^{n_{1}-r_{1}-1}\left(1-q_{1}^{s_{1}} x\right) \prod_{s_{2}=0}^{n_{2}-r_{2}-1}\left(1-q_{2}^{s_{2}} y\right) .
\end{aligned}
$$

We obtain (2.4). The positivity and linearity of $B_{n_{1}, n_{2}}$ follow from relation (2.4).

Lemma 2.5. The generalized bivariate Bernstein operator (2.4) interpolates the function $f$ in the four corners of the unit square, i.e.,

$$
\begin{cases}B_{n_{1}, n_{2}}(f ; 0,0)=f(0,0) ; & B_{n_{1}, n_{2}}(f ; 0,1)=f(0,1) \\ B_{n_{1}, n_{2}}(f ; 1,0)=f(1,0) ; & B_{n_{1}, n_{2}}(f ; 1,1)=f(1,1) .\end{cases}
$$

Proof. We apply lemma 2.4 .

Lemma 2.6. Let $e_{i j}: I^{2} \rightarrow I^{2}, e_{i j}(x, y)=x^{i} y^{j} \quad(0 \leq i+j \leq 2, i, j$ - integers $)$ be the test functions. Then, the following equalities

(i) $\quad B_{n_{1}, n_{2}}\left(e_{00} ; x, y\right)=e_{00}(x, y)$;

(ii) $\quad B_{n_{1}, n_{2}}\left(e_{10} ; x, y\right)=e_{10}(x, y)$;

(iii) $\quad B_{n_{1}, n_{2}}\left(e_{01} ; x, y\right)=e_{01}(x, y)$;

(iv) $\quad B_{n_{1}, n_{2}}\left(e_{11} ; x, y\right)=e_{11}(x, y)$;

(v) $\quad B_{n_{1}, n_{2}}\left(e_{20} ; x, y\right)=e_{20}(x, y)+\frac{x(1-x)}{\left[n_{1}\right]}$

(vi) $B_{n_{1}, n_{2}}\left(e_{02} ; x, y\right)=e_{02}(x, y)+\frac{y(1-y)}{\left[n_{2}\right]}$, 
hold for any $(x, y) \in I^{2}$.

Proof. We show only the proof of (vi). By definition (2.4), one has

$$
\begin{gathered}
B_{n_{1}, n_{2}}\left(e_{02} ; x, y\right)= \\
=\sum_{r_{1}=0}^{n_{1}} \sum_{r_{2}=0}^{n_{2}}\left(\frac{\left[r_{2}\right]}{\left[n_{2}\right]}\right)^{2}\left[\begin{array}{l}
n_{1} \\
r_{1}
\end{array}\right]\left[\begin{array}{c}
n_{2} \\
r_{2}
\end{array}\right] \prod_{s_{1}=0}^{n_{1}-r_{1}-1}\left(1-q_{1}^{s_{1}} x\right) \prod_{s_{2}=0}^{n_{2}-r_{2}-1}\left(1-q_{2}^{s_{2}} x\right)= \\
=\left\{\sum_{r_{1}=0}^{n_{1}}\left[\begin{array}{c}
n_{1} \\
r_{1}
\end{array}\right] x^{r_{1}} \prod_{s_{1}=0}^{n_{1}-r_{1}-1}\left(1-q_{1}^{s_{1}} x\right)\right\}\left\{\sum_{r_{2}=0}^{n_{2}}\left(\frac{\left[r_{2}\right]}{\left[n_{2}\right]}\right)^{2} y^{n_{2}} \prod_{s_{2}=0}^{n_{2}-r_{2}-1}\left(1-q_{2}^{s_{2}} y\right)\right\}= \\
=B_{n_{1}}\left(e_{0} ; x\right) B_{n_{2}}\left(e_{2} ; y\right),
\end{gathered}
$$

where $B_{n_{1}}, B_{n_{2}}$ are the generalized Bernstein operators introduced by G. M. Phillips - see [7] - and $e_{i}: I \rightarrow I, e ;(x)=x^{i}(i=0,1,2)$ are the one variate test functions. It is well known [7] that these operators satisfy the following equalities

$$
B_{n_{1}}\left(e_{0} ; x\right)=e_{0}(x), B_{n_{2}}\left(e_{2} ; y\right)=e_{2}(y)+\frac{y(1-y)}{\left[n_{2}\right]}
$$

for all $x \in I$ and $y \in I$. By using these two equalities, we get (vi).

Theorem 2.1. Let be $q_{1}=q_{1}\left(n_{1}\right), q_{2}=q_{2}\left(n_{2}\right)$, and let $q_{1}\left(n_{1}\right) \rightarrow 1, q_{2}\left(n_{2}\right) \rightarrow 1$ from below as $n_{1} \rightarrow \infty, n_{2} \rightarrow \infty$. Then, for any $f \in C\left(I^{2}\right)$ the sequence of bivariate generalized Bernstein polynomials defined at (2.4) converges uniformly to $f(x, y)$ on $I^{2}$.

Proof. By using relation (1.2) and the hypothesis that $q_{1}=q_{1}\left(n_{1}\right) \rightarrow 1$ as $n_{1} \rightarrow \infty$, one obtains that $\left[n_{1}\right] \rightarrow \infty$ as $n_{1} \rightarrow \infty$.

In a similar way, $\left[n_{2}\right] \rightarrow \infty$ as $n_{2} \rightarrow \infty$.

Next, using lemma 2.6, it follows that $B_{n_{1}, n_{2}}\left(e_{i j} ; x, y\right) \Rightarrow e_{i j}$, uniformly on $I^{2}$ as $n_{1}, n_{2} \rightarrow \infty$.

Applying now the well-known test theorem of Korovkin for bivariate functions, one obtains that $B_{n_{1}, n_{2}}(f ; x, y)$ converges to $f(x, y)$, uniformly on $I^{2}$, and the proof ends.

Theorem 2.2. For any $f: I^{2} \rightarrow \mathbf{R}$, bounded on $I^{2}$, the inequality

$$
\left\|f-B_{n_{1}, n_{2}}(f)\right\|_{\infty} \leq \frac{9}{4} \omega\left(\frac{1}{\sqrt{\left[n_{1}\right]}}, \frac{1}{\sqrt{\left[n_{2}\right]}}\right)
$$

holds.

In (2.5), $\|\cdot\|_{\infty}$ denotes the uniform norm and $\omega$ denotes the first order modulus of smoothness.

Proof. Let $f: I^{2} \rightarrow \mathbf{R}$ be a bivariate bounded function and denoted and let the set of all bounded bivariate functions defined on $\mathbf{I}^{2}$ be denoted by $\mathbf{R}^{I^{2}}$ Then, the 
first order modulus of smoothness is the function $\omega: \mathbf{R}_{+}^{2} \rightarrow[0,1]$, defined for any $f \in \mathbf{R}^{I^{2}}$ and any $\left(\delta_{1}, \delta_{2}\right) \in \mathbf{R}_{+}^{2}$ by

$\omega\left(\delta_{1}, \delta_{2}\right)=\sup \left\{\left|f(x, y)-f\left(x^{\prime}, y^{\prime}\right)\right|(x, y) \in I^{2},\left(x^{\prime}, y^{\prime}\right) \in I^{2},\left|x-x^{\prime}\right|<\delta_{1},\left|y-y^{\prime}\right|<\delta_{2}\right\}$

It is well known that $\omega$, defined at (2.6), is a monotone increasing function with respect to the natural order of $\mathbf{R}^{2}$, i.e.

$$
\left(\delta_{1}, \delta_{2}\right) \in \mathbf{R}_{+}^{2},\left(\delta_{1}^{\prime}, \delta_{2}^{\prime}\right) \in \mathbf{R}_{+}^{2}, \delta_{1}<\delta_{1}^{\prime}, \delta_{2}<\delta_{2}^{\prime} \Rightarrow \omega\left(\delta_{1}, \delta_{2}\right) \leq \omega\left(\delta_{1}^{\prime}, \delta_{2}^{\prime}\right) .
$$

After this introduction, let us to begin the proof. Using lemma (2.6) (the equality (i)), we can write

$$
\begin{gathered}
\left|B_{n_{1}, n_{2}}(f ; x, y)-f(x, y)\right|= \\
=\sum_{r_{1}=0}^{n_{1}} \sum_{r_{2}=0}^{n_{2}}\left[\begin{array}{c}
n_{1} \\
r_{1}
\end{array}\right]\left[\begin{array}{c}
n_{2} \\
r_{2}
\end{array}\right] x^{r_{1}} y^{r_{2}} \times \\
\prod_{s_{1}=0}^{n_{1}-r_{1}-1}\left(1-q_{1}^{s_{1}} x\right) \prod_{s_{2}=0}^{n_{2}-r_{2}-1}\left(1-q_{2}^{s_{2}} y\right)\left(f\left(\frac{\left[r_{1}\right]}{\left[n_{1}\right]}, \frac{\left[r_{2}\right]}{\left[n_{2}\right]}\right)-f(x, y)\right) \leq \\
\leq \sum_{r_{1}=0}^{n_{1}} \sum_{r_{2}=0}^{n_{2}}\left[\begin{array}{c}
n_{1} \\
r_{1}
\end{array}\right]\left[\begin{array}{c}
n_{2} \\
r_{2}
\end{array}\right] x^{r_{1}} y^{r_{2}} \times \\
\prod_{s_{1}=0}^{n_{1}-r_{1}-1}\left(1-q_{1}^{s_{1}} x\right) \prod_{s_{2}=0}^{n_{2}-r_{2}-1}\left(1-q_{2}^{s_{2}} y\right)\left|f\left(\frac{\left[r_{1}\right]}{\left[n_{1}\right]}, \frac{\left[r_{2}\right]}{\left[n_{2}\right]}\right)-f(x, y)\right| .
\end{gathered}
$$

Taking into account the monotonicity of $\omega$, one obtains

$$
\begin{gathered}
\left|f \frac{\left[r_{1}\right]}{\left[n_{1}\right]}, \frac{\left[r_{2}\right]}{\left[n_{2}\right]}-f(x, y)\right| \leq \omega\left(\left|\frac{\left[r_{1}\right]}{\left[n_{1}\right]}-x\right|,\left|\frac{\left[r_{2}\right]}{\left[n_{2}\right]}-y\right|\right) \leq \\
\leq\left(\left|\frac{\left[r_{1}\right]}{\left[n_{1}\right]}-x\right| \sqrt{\left[n_{1}\right]}+1\right)\left(\left|\frac{\left[r_{2}\right]}{\left[n_{2}\right]}-y\right| \sqrt{\left[n_{2}\right]}+1\right) \omega\left(\frac{1}{\sqrt{\left[n_{1}\right]}}, \frac{1}{\sqrt{\left[n_{2}\right.}}\right)
\end{gathered}
$$

From (2.7), (2.8) follows the inequality

$$
\left|B_{n_{1}, n_{2}}(f ; x, y)-f(x, y)\right| \leq K
$$

where

$$
\begin{gathered}
K=\omega\left(\frac{1}{\sqrt{\left[n_{1}\right]}}, \frac{1}{\sqrt{\left[n_{2}\right.}}\right) \times \\
\left(\sqrt{\left[n_{1}\right]} \sum_{r_{1}=0}^{n_{1}}\left|\frac{\left[r_{1}\right]}{\left[n_{1}\right]}-1\right|\left[\begin{array}{c}
n_{1} \\
r_{1}
\end{array}\right] x^{r_{1}} \prod_{s_{1}=0}^{n_{1}-r_{1}-1}\left(1-q_{1}^{s_{1}} x\right)+1\right) \times \\
\left(\sqrt{\left[n_{2}\right]} \sum_{r_{2}=0}^{n_{2}}\left|\frac{\left[r_{2}\right]}{\left[n_{2}\right]}-1\right|\left[\begin{array}{c}
n_{2} \\
r_{2}
\end{array}\right] y^{r_{2}} \prod_{s_{2}=0}^{n_{2}-r_{2}-1}\left(1-q_{2}^{s_{2}} y\right)+1\right) .
\end{gathered}
$$


Next, applying the Schwarz inequality, one obtains

$$
\begin{aligned}
& \left(\sum_{r_{1}=0}^{n_{1}}\left(\frac{\left[r_{1}\right]}{\left[n_{1}\right]}-x\right)\left[\begin{array}{c}
n_{1} \\
r_{1}
\end{array}\right] x \prod_{s_{1}=0}^{r_{1}}\left(1-q_{1}^{s_{1}} x\right)\right)^{2}= \\
= & \sum_{r_{1}=0}^{n_{1}}\left|\frac{\left[r_{1}\right]}{\left[n_{1}\right]}-x\right|\left(\left[\begin{array}{c}
n_{1} \\
r_{1}
\end{array}\right] x^{r_{1}} \prod_{s_{1}=0}^{n_{1}-r_{1}-1}\left(1-q_{1}^{s_{1}} x\right)\right)^{\frac{1}{2}} \times \\
& \left.\left(\left[\begin{array}{c}
n_{1} \\
r_{1}
\end{array}\right] x^{r_{1}} \prod_{s_{1}=0}^{n_{1}-r_{1}-1}\left(1-q_{1}^{s_{1}} x\right)\right)^{\frac{1}{2}}\right)^{2} \leq \\
\leq & \left(\sum_{r_{1}=0}^{n_{1}}\left(\frac{\left[r_{1}\right]}{\left[n_{1}\right]}-x\right)^{2}\left[\begin{array}{c}
n_{1} \\
r_{1}
\end{array}\right] x^{r_{1}} \prod_{s_{1}=0}^{n_{1}-r_{1}-1}\left(1-q_{1}^{s_{1}} x\right)\right) \times \\
= & \left\{B_{n_{1}}\left(e_{2} ; x\right)-2 x \cdot B_{r_{1}=0}\left[\begin{array}{c}
n_{1} \\
r_{1}
\end{array}\right] x^{r_{1}} \prod_{n_{1}=0}^{n_{1}-r_{1}-1}\left(1-q_{1}^{s_{1}} x\right)\right)= \\
= & x^{2}+\frac{x(1-x)}{\left[n_{1}\right]}-2 x^{2}+x^{2}=\frac{x(1-x)}{\left[n_{1}\right]} \leq \frac{1}{4\left[n_{1}\right]} .
\end{aligned}
$$

In a similar way we obtain

$$
\left(\sum_{r_{2}=0}^{n_{2}}\left(\frac{\left[r_{2}\right]}{\left[n_{2}\right]}-y\right)\left[\begin{array}{c}
n_{2} \\
r_{2}
\end{array}\right] y^{r_{2}} \prod_{s_{2}=0}^{n_{2}-r_{2}-1}\left(1-q_{2}^{s_{2}} y\right)\right)^{2} \leq \frac{1}{4\left[n_{2}\right]} .
$$

Combining now the inequalities (2.9), (2.10) and (2.11), one obtains

$$
\begin{aligned}
& \left|B_{n_{1}, n_{2}}(f ; x, y)-f(x, y)\right| \leq \omega\left(\frac{1}{\sqrt{\left[n_{1}\right]}}, \frac{1}{\sqrt{\left[n_{2}\right]}}\right) \times \\
& \quad\left(\sqrt{\left[n_{1}\right]} \cdot \frac{1}{2 \sqrt{\left[n_{1}\right]}}+1\right)\left(\sqrt{\left[n_{2}\right]} \cdot \frac{1}{2 \sqrt{\left[n_{2}\right]}}+1\right)=\frac{9}{4} \omega\left(\frac{1}{\sqrt{\left[n_{1}\right]}}, \frac{1}{\sqrt{\left[n_{2}\right]}}\right)
\end{aligned}
$$

From (2.12), computing the supremum follows (2.5) and the proof ends.

\section{Remarks 2.1.}

(i) Clearly, if $q_{1}=q_{2}=1$, then operator (2.4) is reduced to the classical Bernstein bivariate operator, considered first by D. D. Stancu [10].

In this case, the results contained in theorems 2.1 and 2.2 are reduced to the well-known results due to D. D. Stancu [10,11].

(ii) If $q_{1}=1, q_{2} \neq 1$ or $q_{1} \neq 1, q_{2}=1$, other interesting operators of Bernstein type can be obtained. The approximation properties of these operators are similar to the properties of the classical bivariate operator of Bernstein. 
(iii) All the results contained in the present paper can be extended to the case of n-variate functions.

Finally, we mention that a first form of this paper was presented at " microCAD 99" (in Miskolc, Hungary, February 1999), and we express our gratitude to all our colleagues from Miskolc, especially to Professor Aurél Galántai.

\section{REFERENCES}

[1] Badea, C., Badea, I. and Gonska H.H.: A test function theorem and approximation by pseudopolynomials, Bul. Australl.Math. Soc., 34, (1986), 53-64.

[2] Barbosu, D.: The approximation of the Bogel- continuous functions using the BernsteinStancu polynomials, Bul. Ştiinț. Univ. Baia Mare, seria B, Mat-Inf, Vol. VIII, (1992), 11-18.

[3] Barbosu, D.: On a blending operator Bernstein-Stancu type, Proceed. of the Annual Conference of the Romanian Society of Mathematical Sciences 1997, May 29-June 1, Bucharest Romania, 217-222.

[4] Barbosu, D.: Bivariate Bernstein operators with knots in geometric progression, (to appear in "Proceed. of microCAD'99", International Computer Science Conference in Miskolc, Hungary.)

[5] Delvos, F. I. and Schemp, W.: Boolean Methods in Interpolation and Approximation, Longman Scientific \& Techical, 1989.

[6] Dobrescu, E. and Matei, I.: Aproximarea prin polinoame de tip Bernstein a functiilor bidimensional continue, Anal. Univ. Timişoara, Seria Ştiinţe Matematice-Fizice, Vol. IV, (1966), 85-90. (in Romanian)

[7] Phillips, G. M.: On generalized Bernstein polynomials, Numerical Analysis: A. R. Mitchell $75^{\text {th }}$ Birthday Volume, World Scientific, Singapore, 1996.

[8] Phillips, G. M.: Bernstein polynomials based on the q-integers, Festschrift for T.J.Rivlin, Baltzer, New-York, 1996. (to appear)

[9] Phillips, G. M.: On generalized Bernstein polynomials, Proceed. of I.C.A.O.R, ClujNapoca, July 29-August 1, 1996, Vol. I, Transilvania Press Cluj-Napoca, Romania, 1997, 335-340.

[10] Stancu D. D.: A new class of uniform approximating polynomial operators in two and several variables, Proceed. of the Conference on Constructive Theory of Functions, Budapest (1969), 31-45.

[11] Stancu D. D.: Approximation properties of a class of linear positive operators, Studia Univ. "Babeş-Bolyai", Cluj-Napoca, Mathematica, 15, (1970), 33-38.

[12] Stancu D. D.: Approximation of functions by means of some new classes of positive linear operators, Proceed. Conf. Oberwolfach (1971), "Numerische Methoden der Approximation Theorie", Bd.1, ISNN 16, Basel (1972), 187-203. 